\title{
글
}

CLINICAL RESEARCH

\section{Effects of mouth rehabilitation with removable complete dentures on stimulus perception and the electromyographic activity of the orbicularis oris muscle}

\author{
Fernanda P. de Caxias, DDS, MS, ${ }^{a}$ Daniela M. dos Santos, MS, PhD, ${ }^{b}$ Marcelo C. Goiato, MS, PhD, ${ }^{c}$ \\ Sandro B. Bitencourt, DDS, MS, ${ }^{d}$ Emily V. F. da Silva, DDS, MS, ${ }^{e}$ Murilo C. B. Laurindo-Junior, DDS, ${ }^{f}$ and \\ Karina H. L. Turcio, MS, $\mathrm{PhD}^{\mathrm{g}}$
}

Increased life expectancy ${ }^{1}$ has led researchers to study aging and its characteristics, the physiological and psychological attributes of the elderly, and the effects of mouth rehabilitation on the stomatognathic system. Some elderly individuals have difficulty performing oral hygiene, which leads to periodontal disease and caries that result in high tooth loss rates. ${ }^{2}$ Modern dentistry prioritizes rehabilitation treatment with implants. However, the use of a removable complete denture is the only rehabilitation option for many patients because of their systemic health, ${ }^{3,4}$ alveolar bone characteristics, ${ }^{5}$ or financial situation. Although removable complete dentures are not the gold standard for rehabilitating patients with edentulism, when well-made and well-adapted,

\begin{abstract}
Statement of problem. Many elderly individuals are rehabilitated with removable complete dentures, which require an initial adaptation period for both oral perception and the perioral muscles. Studies assessing the changes in stimulus perception and the electromyographic (EMG) activity of the orbicularis oris muscle shortly after conventional complete denture insertion are lacking.
\end{abstract}

Purpose. The purpose of this clinical study was to evaluate the effect of mouth rehabilitation with removable complete dentures on stimulus perception and the EMG activity of the orbicularis oris muscle.

Material and methods. This study was approved by the Human Research Ethics Committee of the Araçatuba Dental School (São Paulo State University). Fifteen participants who had worn their removable complete dentures for at least 5 years and needed rehabilitation with new prostheses were enrolled in the study. A perception questionnaire was applied, and surface EMG examinations of the orbicularis oris muscle during rest, suction of water with a straw, and pronunciation of the syllables /bah/, /mah/, /pah/, and the word 'Mississippi' were performed before (T0) and 30 (T1) and 100 (T2) days after insertion of the new prostheses. The data were analyzed with the Cochran $\mathrm{Q}$ test, McNemar test, 2-way repeated measures ANOVA, and honestly significant difference (HSD) Tukey test $(\alpha=.05)$

Results. Significant improvement was reported in the perception questionnaire in terms of the oral discomfort sensation in the T2 period. EMG activity decreased during rest and suction after insertion of the new prostheses. A statistical difference between the upper and lower fascicles of the orbicularis oris muscle was detected, with a decrease of EMG activity between the T0 and T1 periods on the lower fascicle, except for when pronouncing the /pah/ syllable.

Conclusions. Mouth rehabilitation with removable complete dentures decreased oral discomfort and, depending on the oral function, decreased or increased EMG activity of the orbicularis oris muscle. In addition, the lower fascicle was more active than the upper fascicle during rest and most functional activities. (J Prosthet Dent 2018;119:749-54)

\footnotetext{
aPostgraduate student, Department of Dental Materials and Prosthodontics, São Paulo State University, School of Dentistry, São Paulo, Brazil.

bProfessor, Department of Dental Materials and Prosthodontics, São Paulo State University, School of Dentistry, São Paulo, Brazil.

cProfessor, Department of Dental Materials and Prosthodontics, São Paulo State University, School of Dentistry, São Paulo, Brazil.

${ }^{d}$ Postgraduate student, Department of Dental Materials and Prosthodontics, São Paulo State University, School of Dentistry, São Paulo, Brazil.

ePostgraduate student, Department of Dental Materials and Prosthodontics, São Paulo State University, School of Dentistry, São Paulo, Brazil.

fPostgraduate student, Department of Dental Materials and Prosthodontics, São Paulo State University, School of Dentistry, São Paulo, Brazil.

gProfessor, Department of Dental Materials and Prosthodontics, São Paulo State University, School of Dentistry, São Paulo, Brazil.
} 


\section{Clinical Implications}

Knowledge of the electromyographic activity of the orbicularis oris muscles shortly after insertion of removable complete dentures and of stimulus perception related to these prostheses offers the professional a scientific basis for educating patients to accept the difficulties of adaptation as one of the rehabilitation treatment phases.

they provide favorable esthetics and good retention and stability, ${ }^{6}$ all of which improve patient masticatory efficiency ${ }^{7}$ and consequently quality of life. ${ }^{8}$

Proprioceptors in the oral cavity, ${ }^{9}$ muscles, and joints receive stimuli ${ }^{10}$ and present sensory responses ${ }^{9}$ with mechanisms that exhibit important differences in the elderly..$^{10}$ Although orofacial sensitivity tends to decrease with aging, ${ }^{11}$ patients can experience oral discomfort, mainly after the insertion of new prostheses, the feeling of a "full mouth," difficulty pronouncing the sibilants, and excessive salivation. ${ }^{8}$ The fingers, followed by the mouth (principally the tongue and lips), are areas that have the greatest tactile sensitivity, ${ }^{12}$ reinforcing the need for a well-adapted prosthesis to avoid patient discomfort and allow for a short adaptation period. The lip muscles, principally the orbicularis oris, function with the cheek muscles to create contact between the prosthetic teeth and the lips by pressurizing the dental arches ${ }^{13}$ and collaborating in the production of speech sounds. ${ }^{13,14}$ The orbicularis muscle is also important in swallowing, ${ }^{15,16}$ mastication, and sucking. ${ }^{16}$ Muscular behavior can be studied with electromyography, ${ }^{17-19}$ which can measure the intensity and duration of muscular activity and also identify characteristics associated with fatigue. ${ }^{20}$

Studies of orbicularis oris muscle behavior can be found dating back more than 50 years, ${ }^{21-23}$ but few have investigated the behavior of this muscle in individuals with complete dentures ${ }^{13,24,25}$ The authors are unaware of studies comparing the electromyographic (EMG) activity of that muscle or stimulus perception before or during the initial adaptation period after the insertion of complete dentures. Because the orbicularis oris muscle participates in different oral functions ${ }^{26}$ such as speech, suction, and lip projection (which occurs during kissing), ${ }^{16}$ studying EMG activity is a reliable method of evaluating its behavior. ${ }^{13,24}$ The lips are moved by the orbicularis oris muscle but also depend on muscles such as the lower lip depressor and the risorius muscle. ${ }^{27}$

The purpose of this study was to analyze stimulus perception and the EMG activity of the orbicularis oris muscle of removable complete denture wearers, with their old prostheses and after the insertion of a new removable complete denture. The null hypothesis was that rehabilitation with new removable complete dentures would not influence stimulus perception or the EMG activity of the orbicularis oris muscle, regardless of the evaluation period.

\section{MATERIAL AND METHODS}

Participants in this study were patients from the Complete Denture Clinic of the Aracatuba Dental School, São Paulo State University (UNESP), and from the Prosthesis Specialization Course Clinic of the São Paulo DentalSurgeons Association. The inclusion criteria were individuals with edentulism who, according to the Prosthodontic Diagnostic Index Complete Edentulism Checklist, ${ }^{28}$ were classified in all classes, except for "limited interarch space" (class III only), and showed no "conditions requiring preprosthetic surgery" or "modifiers." Patients who had been using the same prostheses for at least 5 consecutive years, who had normal health or mild systemic disease, ${ }^{29}$ and who had sufficient cognitive ability and comprehension to answer the questions asked and to follow the instructions given were also included. In addition, they must have been using unsatisfactory prostheses presenting a loss of occlusal vertical dimension (OVD), occlusal wear, instability, and poor retention, and in need of new bimaxillary removable complete dentures. Individuals who reported neurological or psychiatric disorders, who were using psychotherapeutic agents, or who had history of a tumor or a temporomandibular disorder confirmed by the Research Diagnostic Criteria ${ }^{30}$ questionnaire were excluded.

Demographic data were collected by using the Research Diagnostic Criteria questionnaire. The selected participants were given verbal and written information about the purpose of the study and were requested to sign an informed consent form in accordance with the Ethics Committee recommendations (Aracatuba Dental School, UNESP). They were given a copy signed by the responsible researchers (K.H.L., D.M.S.). This study was approved by the Ethics Committee of the Aracatuba Dental School-UNESP under opinion number 1.165.721/ 2015. Clinical data for the study were collected in the following sequence: evaluations of stimulus perception by using a perception questionnaire ${ }^{8}$ and EMG examinations of the orbicularis oris muscle while the participant was still wearing the existing prostheses (maxillary and mandibular), before insertion of the new prostheses (T0). All the procedures were repeated at 30 (T1) and 100 (T2) days after insertion of the new prostheses.

The perception questionnaire was composed of 4 questions that evaluated the feeling of a "full mouth" (question 1), pronunciation of sibilants (question 2), discomfort with the prostheses (question 3), and excessive salivation (question 4) (Table 1). Participants were 
Table 1. Frequency and percentage of answers to each question of perception questionnaire $(n=15)$

\begin{tabular}{|c|c|c|c|c|c|c|c|}
\hline \multirow[b]{3}{*}{ Question } & \multicolumn{6}{|c|}{ Period } & \multirow[b]{3}{*}{$\boldsymbol{P}$} \\
\hline & \multicolumn{2}{|c|}{ TO } & \multicolumn{2}{|c|}{ T1 } & \multicolumn{2}{|c|}{ T2 } & \\
\hline & No & Yes & No & Yes & No & Yes & \\
\hline Do you have the feeling of a "full mouth"? & 12 & $3(20 \%)$ & 7 & $8(53.3 \%)$ & 8 & $7(46.7 \%)$ & .097 \\
\hline Do you have any difficulty pronouncing sibilants? & 9 & $6(40 \%)$ & 7 & $8(53.3 \%)$ & 10 & $5(33.3 \%)$ & .417 \\
\hline Do you feel any discomfort with the prosthesis? & 8 & $7(46.7 \%)$ & 4 & $11(73.3 \%)$ & 11 & $4(26.7 \%)$ & $.016^{*}$ \\
\hline Are you salivating excessively? & 9 & $6(40 \%)$ & 11 & $4(26.7 \%)$ & 10 & 5 (33.3\%) & .687 \\
\hline
\end{tabular}

given only 2 answer options: no=0 and yes $=1$. The questions were read to the patients by only 1 examiner (F.P.C.), because not all participants were able to read them.

For the EMG examinations, a device (MyosystemBr1; DataHominis Tecnologia Ltd) and software were used to visualize and process the EMG signal. Dischargeable double-surface electrodes made of polyethylene foam with medical adhesive, silver/silver chloride $(\mathrm{Ag} / \mathrm{AgCl})$ double contact, and adherent hydrogel with low impedance (Hal Industria \& Comercio Ltd) were used to record the EMG signal. A ground electrode was positioned $^{31}$ on the wrist of the participant to minimize any possible interference. All examinations were performed by the same operator (F.P.C.). Before the placement of the electrodes, the faces of the participants were washed with water and astringent soap. ${ }^{32}$ Oil from the skin where the electrodes were to be placed was removed by gentle rubbing with cotton and $70 \%$ alcohol to reduce impedance and improve signal conductivity. ${ }^{31,32}$

A total of 4 electrodes were positioned above and below the vermillion border of the lips, with each positioned longitudinally to the muscle bundle on the right and left side of both lips. Signals were recorded during mandibular rest (10 seconds), ${ }^{33}$ suction of water with a straw $^{32}$ (10-cm length $\times 3-\mathrm{mm}$ thickness) (6 seconds), and pronunciation of the syllables $/ \mathrm{bah} /, / \mathrm{mah} /$, and $/ \mathrm{pah} /$ and the word 'Mississippi' 24 (3 times each, after which the highest value was tabulated). During the examinations, the participants were seated on a chair with their feet on the floor and their arms on their lap, ${ }^{24}$ and the Frankfurt plane (parallel to the floor) was used as a reference to keep the head straight. ${ }^{24}$ All complete prostheses were fabricated according to the technique recommended by Zarb et al. ${ }^{34}$

The perception questionnaire was evaluated by using binary answers (no=0 and yes=1). The data were submitted to statistical analysis with the Cochran $Q$ test $(\alpha=.05)$ to evaluate the frequency and percentage of each question, as well as the variation among the 3 periods studied (T0, T1, and T2). The McNemar test was used to compare the differences among the periods. Software was used to determine the root mean square (RMS) value of the electrical signal $(\mu \mathrm{V})$ obtained during the EMG examinations. The arithmetic means were calculated for the right and left sides of each fascicle. The EMG data obtained during rest, suction, and phonetic testing were submitted to a 2-way repeated-measures ANOVA. Subsequently, the data from all tests were submitted to the honestly significant difference (HSD) Tukey test $(\alpha=.05)$. The Pearson correlation test was used to assess the correlation between question 1 (feeling of a "full mouth") and EMG activity during rest and between question 2 (difficulty pronouncing sibilants) and EMG activity during pronunciation of the word 'Mississippi.'

\section{RESULTS}

Twenty-two participants were enrolled in the study after meeting the inclusion criteria. Of these, 7 left the study before its conclusion due to personal reasons or because they did not wear their prostheses or had health problems. Therefore, 15 participants (12 women and 3 men) with a mean age of 65 years (range: 51 to 87 years) were examined by EMG and answered the perception questionnaire. The existing prostheses were worn by the participants for a mean of 17 years (range: 5 to 48 years) according to the participant reports.

The results of the perception questionnaire are presented in Table 1. A statistical difference was found for the oral discomfort sensation in the T2 period ( $P=.016$ Cochran Q test, T0, T1, and T2). This difference was confirmed by the McNemar test $(P=.016)$ when periods $\mathrm{T} 1$ and $\mathrm{T} 2$ were compared.

The results of 2-way ANOVA tests determined that EMG activity values were influenced by period during rest $(\mathrm{df}=2 ; \mathrm{F}=3.874 ; P=.027)$ and suction $(\mathrm{df}=2 ; \mathrm{F}=3.506$; $P=.037$ ), and they were influenced by analysis region during the pronunciation of $/ \mathrm{bah} /(\mathrm{df}=1 ; \mathrm{F}=22.244$; $P<.001), \quad / \mathrm{mah} /(\mathrm{df}=1 ; \mathrm{F}=22.866 ; P<.001), \quad / \mathrm{pah} / \quad(\mathrm{df}=1 ;$ $\mathrm{F}=22.963 ; \quad P<.001)$, and 'Mississippi' $(\mathrm{df}=1 ; \mathrm{F}=25.903$; $P<.001)$. During rest, a difference was detected between periods T0 and T2 $(P<.05)$ in the upper fascicle of the orbicularis oris muscle, with higher EMG activity in period T0 (Table 2). During suction, EMG activity values were higher in the lower fascicle when compared with the upper fascicle in the T0 period $(P<.05)$. EMG activity in both the upper and lower fascicles was higher in the T1 period when compared with T2 $(P<.05)$ (Table 3). Numerically similar values were observed for the upper 
Table 2. RMS mean values \pm standard deviation of electromyographic activity $(\mu \mathrm{V})$ of orbicularis oris muscle for each region of analysis (upper and lower) during rest

\begin{tabular}{lccc}
\hline & \multicolumn{3}{c}{ Period } \\
\cline { 2 - 4 } Analysis Region & T0 & T1 & T2 \\
\hline Upper & $9.69 \pm 4.28^{\mathrm{Aa}}$ & $7.80 \pm 2.90^{\mathrm{Aab}}$ & $6.90 \pm 2.48^{\mathrm{Ab}}$ \\
\hline Lower & $10.43 \pm 8.24^{\mathrm{Aa}}$ & $10.44 \pm 7.07^{\mathrm{Aa}}$ & $8.66 \pm 5.10^{\mathrm{Aa}}$ \\
\hline
\end{tabular}

Means followed by same superscript uppercase letter in column and lowercase letter in row not significantly different (P>.05) with HSD Tukey test.

Table 4. RMS mean values (standard deviation) of electromyographic activity $(\mu \mathrm{V})$ of orbicularis oris muscle for each region of analysis (upper and lower) during phonetic tests

\begin{tabular}{|c|c|c|c|c|c|c|c|}
\hline \multirow[b]{3}{*}{ EMG } & \multirow{3}{*}{$\begin{array}{l}\text { Analysis } \\
\text { Region }\end{array}$} & \multicolumn{6}{|c|}{ Period } \\
\hline & & \multicolumn{2}{|c|}{ TO } & \multicolumn{2}{|c|}{ T1 } & \multicolumn{2}{|c|}{ T2 } \\
\hline & & Mean & SD & Mean & SD & Mean & SD \\
\hline \multirow[t]{2}{*}{ /bah/ } & Upper & $32.40^{\mathrm{Aa}}$ & 13.89 & $35.09^{\mathrm{Aa}}$ & 18.11 & $39.69^{\mathrm{Aa}}$ & 15.74 \\
\hline & Lower & $90.52^{\mathrm{Ba}}$ & 54.67 & $76.61^{\mathrm{Bb}}$ & 33.59 & $81.91^{\mathrm{Bab}}$ & 27.93 \\
\hline \multirow[t]{2}{*}{$/ \mathrm{mah} /$} & Upper & $32.32^{\mathrm{Aa}}$ & 14.45 & $33.02^{\mathrm{Aa}}$ & 18.69 & $33.82^{\mathrm{Aa}}$ & 13.79 \\
\hline & Lower & $90.38^{\mathrm{Ba}}$ & 54.85 & $75.84^{\mathrm{Bb}}$ & 33.42 & $76.18^{\mathrm{Bab}}$ & 25.81 \\
\hline \multirow[t]{2}{*}{ /pah/ } & Upper & $35.37^{\mathrm{Aa}}$ & 14.99 & $38.21^{\mathrm{Aa}}$ & 18.30 & $41.14^{\mathrm{Aa}}$ & 16.15 \\
\hline & Lower & $99.90^{\mathrm{Ba}}$ & 60.58 & $85.05^{\mathrm{Ba}}$ & 35.15 & $85.44^{\mathrm{Ba}}$ & 30.30 \\
\hline \multirow[t]{2}{*}{ Mississippi } & Upper & $37.62^{\mathrm{Aa}}$ & 15.19 & $37.76^{\mathrm{Aa}}$ & 20.47 & $40.63^{\mathrm{Aa}}$ & 17.54 \\
\hline & Lower & $123.63^{\mathrm{Ba}}$ & 84.95 & $98.11^{\mathrm{Bb}}$ & 40.28 & $101.33^{\mathrm{Bab}}$ & 37.23 \\
\hline
\end{tabular}

SD, Standard deviation. Means followed by same superscript uppercase letter in column and lowercase letter in row not significantly different $(P>.05)$ with HSD Tukey test.

and lower fascicles in T1 and T2 (Table 3). The EMG activity in the lower fascicle was statistically higher than the upper fascicle during pronunciation of $/ \mathrm{bah} /, / \mathrm{mah} /$, /pah/, and 'Mississippi' (Table 4) in all analysis periods. Higher EMG activity was observed in the lower fascicle in period T0, with a statistical difference $(P<.05)$ from period T1 for the /bah/, /mah/, and 'Mississippi' phonetics. No correlation $(P>.05)$ was found between question 1 and EMG activity during rest (Table 5 ) or between question 2 and EMG activity during pronunciation of the word 'Mississippi' (Table 6).

\section{DISCUSSION}

The null hypothesis that rehabilitation with new removable complete dentures would not influence stimulus perception or the EMG activity of the orbicularis oris muscle, regardless of the evaluation period, was rejected because rehabilitation influenced these characteristics.

The oral discomfort report assessed by using the perception questionnaire, which was the domain that showed improvement at 100 days after new prosthesis insertion $(P=.016)$, seems to be related to the adaptation period of participants to their new prostheses ${ }^{8}$; this may vary according to their muscular ability and capacity. ${ }^{17}$ Among the factors leading to the oral sensations reported by patients after insertion of new conventional complete prostheses are the recovery of the $\mathrm{OVD}_{1}^{8}$ which
Table 3. RMS mean values \pm standard deviation of electromyographic activity $(\mu \mathrm{V})$ of orbicularis oris muscle for each region of analysis (upper and lower) during suction

\begin{tabular}{lccc}
\hline & \multicolumn{3}{c}{ Period } \\
\cline { 2 - 4 } Analysis Region & T0 & T1 & T2 \\
\hline Upper & $42.94 \pm 24.17^{\mathrm{Aab}}$ & $56.07 \pm 42.02^{\mathrm{Aa}}$ & $36.42 \pm 21.70^{\mathrm{Ab}}$ \\
\hline Lower & $84.40 \pm 73.98^{\mathrm{Ba}}$ & $52.88 \pm 23.97^{\mathrm{Aab}}$ & $38.00 \pm 21.93^{\mathrm{Ab}}$ \\
\hline
\end{tabular}

Means followed by same superscript uppercase letter in column and lowercase letter in row not significantly different (P>.05) with HSD Tukey test.

Table 5. Pearson coefficient correlation between answers to question 1 from perception questionnaire (feeling of "full mouth") and EMG activity of orbicularis oris during rest

\begin{tabular}{ccc}
\hline Pearson Correlation & $\begin{array}{c}\text { Answer to } \\
\text { Question 1 }\end{array}$ & $\begin{array}{r}\text { EMG Activity } \\
\text { During Rest }\end{array}$ \\
\hline Answer to Question 1 & & \\
\hline Correlation coefficient & 1.000 & -.131 \\
\hline$P$ & & .219 \\
\hline N & 90 & 90 \\
\hline EMG Activity During Rest & & 1.000 \\
\hline Correlation coefficient & -.131 & \\
\hline$P$ & .219 & 90 \\
\hline N & 90 & \\
\hline
\end{tabular}

EMG, electromyographic. $P<.05$ indicates statistically significant difference.

Table 6. Pearson coefficient correlation between answers to question 2 of perception questionnaire (difficulty pronouncing sibilants) and EMG activity of orbicularis oris during pronunciation of word 'Mississippi'

\begin{tabular}{lcc}
\hline $\begin{array}{l}\text { Pearson } \\
\text { Correlation }\end{array}$ & $\begin{array}{c}\text { Answer to } \\
\text { Question 2 }\end{array}$ & $\begin{array}{c}\text { EMG Activity: Word } \\
\text { 'Mississippi' }\end{array}$ \\
\hline Answer to Question 2 & & \\
\hline Coefficient correlation & 1.000 & -.090 \\
\hline$P$ & & .398 \\
\hline $\mathrm{N}$ & 90 & 90 \\
\hline EMG Activity: Word 'Mississippi' & & 1.000 \\
\hline Coefficient correlation & -.090 & \\
\hline$P$ & .398 & 90 \\
\hline $\mathrm{N}$ & 90 & \\
\hline
\end{tabular}

EMG, electromyographic. $P<.05$ means statistically significant difference.

may have been reduced by progressive occlusal wear of the artificial teeth in the old prostheses, ${ }^{35}$ and the new tooth arrangement. ${ }^{13}$ Additional factors may be a change in the prosthesis base thickness ${ }^{13}$ and the neuronal reflex caused by prosthesis contact with the buccal mucosa, which triggers a sensory response stimulating salivary production. ${ }^{9}$

The decrease of EMG activity in T2 in the upper fascicle between periods $\mathrm{T} 0$ and $\mathrm{T} 2$ (Table 2) during mandibular rest implies that the labial support offered by the new anterior teeth may facilitate muscular function, requiring less effort because it allows the prosthesis to be more comfortable and better adapted to the muscles. ${ }^{24}$ In dentate patients with competent lips, the EMG activity of the orbicularis oris is lower because the muscle exerts less effort to perform the functions studied. ${ }^{23}$ This same 
reason may be the cause of decreased EMG activity found for the upper fascicle during suction (Table 3), but additional studies assessing labial efficiency are required for confirmation.

Rehabilitation improves prosthetic stability and gives individuals confidence ${ }^{8}$ in fulfilling their daily activities. Increased self-esteem ${ }^{8}$ allows the lip muscles to be more relaxed, which is related to the proper restoration of the OVD, which allows reprograming of muscle extension and tone. ${ }^{31}$ In turn, the highest activity found in T0 during rest can be explained by attempts to keep an unstable prosthesis in position, causing muscle contraction and consequently an increase in the EMG electrical activity of the muscle. ${ }^{18}$

During suction, increased electrical activity was found on the inferior fascicle when the patient was using the old prostheses (T0) (Table 3). This may be due to poorly fitting old prostheses, with the mandibular prosthesis generally more unstable due to inadequate bone support. ${ }^{13}$ This makes them less compatible with mandibular movements and functional activities, which causes the muscles to be more active in keeping the prostheses in place. ${ }^{6}$ Associated with this fact is mandibular projection caused by OVD reduction, ${ }^{13}$ which results in the contraction of the perioral muscle on which the suction movement with a straw depends. ${ }^{19}$ A greater effort is required to perform suction movements when an individual has malocclusion, which leads to higher EMG activity. ${ }^{19}$ In the case of complete prosthesis users, malocclusion could correspond to the reduction of OVD. The higher activity found for the upper fascicle at 30 days (T1) may be related to the fact that patients, in addition to the muscular adaptation, were still uncertain about the retention of their new prostheses during the suction movement. ${ }^{7}$ The numerically similar values found for the upper and lower fascicles in periods T1 and T2 suggest that both fascicles are activated with greater intensity during suction when the prosthesis is well-adapted to the oral cavity (Table 3).

For the phonetic tests (Table 4), the values recorded by the upper fascicle were similar in all the tests, with no statistical difference and a slight progressive increase. An increase of EMG activity in the upper fascicle after the insertion of the new prostheses was also reported by Santos et al. ${ }^{13}$ During mandibular rest, better lip support and comfort were offered by the position of the new anterior teeth, consequently lowering EMG activity. However, unlike the rest period, an increase of EMG activity was seen in the phonetic tests. This may be explained by greater base thickness and the new position of the anterior teeth since the interaction of the lips and prostheses can modify muscular behavior. ${ }^{13}$

For all phonetic tests except for pronunciation of the /pah/ syllable, the statistically significant decrease in EMG activity between periods $\mathrm{T} 0$ and $\mathrm{T} 1$ can be explained by the process of adaptation to the prostheses. ${ }^{17}$ This suggests that participants performed smoother movements, probably because they hesitated to speak with the newly inserted prostheses. The smaller values of EMG activity in T1 and T2 when compared with T0 differed from those found by Santos et $\mathrm{al}^{13}{ }^{13}$ who reported an increase in this activity for most phonetic testing when the participants wore the new prostheses. This difference may be related to the elapsed time between the insertion of the new prostheses or the EMG analysis, because these authors evaluated their patients 5 months after rehabilitation, whereas in the present study evaluation occurred 30 (T1) and 100 days (T2) after insertion of the new prostheses. EMG activity in the present study tended to change after 100 days, as the patients adapted to the prostheses and felt more confident with speech.

In all EMG evaluations, except in period T1 during suction (Table 3), the inferior fascicle values were found to be numerically higher than those in the upper fascicle. This was not unexpected and indicates that the inferior fascicle is more active. Other studies with different clinical situations have reported similar findings. ${ }^{13,16,24,25}$ The variation in EMG activity between the upper and lower portions of the orbicularis oris muscle may be explained by the intramuscular and interindividual variation in the fiber pattern ${ }^{26}$ and the fact that the mentalis muscle works synergistically with the inferior fasciculus of the orbicularis oris muscle during labial sealing, ${ }^{22}$ contributing perhaps to greater mobility and consequent higher EMG activity. Furthermore, in the present study, prosthetic instability was specified as the reason for contraction of the lower lip and consequent higher EMG activity. Prosthesis stability and retention depend on factors such as surface tension, salivary viscosity, adaptation of the base to the residual ridge, peripheral sealing, settling force, and soft tissue adaptation. ${ }^{6}$ The difficulty of achieving these factors in the mandible, added to bone resorption in the anterior region, which causes projection of the mentalis muscle, ${ }^{4}$ hamper stability of the prosthesis.

This study assessed only the correlations between question 1 and the EMG activity during rest and between question 2 and EMG activity during pronunciation of the word 'Mississippi' because these are the 2 domains on the perception questionnaire that seemed related to rest and the sibilants present in the word 'Mississippi.' New studies with larger samples are needed to evaluate the absence of correlation found in the present study.

A limitation of this study is the absence of a control group (for example, patients who would not receive new dentures), which would allow comparison between rehabilitated and nonrehabilitated patients. Future studies should compare both clinical situations to evaluate whether these changes occur in the absence of new dentures and also compare stimulus perception and the 
EMG activity of the orbicularis oris muscle in patients with removable complete dentures and with implantsupported overdentures.

\section{CONCLUSIONS}

Based on the results of this clinical study, the following conclusions were drawn:

1. The oral discomfort sensation was reduced 100 days after insertion of the new prostheses.

2. EMG activity decreased in the upper fascicle of the orbicularis oris muscle during rest.

3. EMG activity in the upper fascicle increased at 30 days, followed by a decrease at 100 days and a decrease in the upper fascicle during suction at 100 days.

4. EMG activity in the inferior fascicle decreased during the phonetic tests at 30 days, except for during pronunciation of the / pah/ syllable. In addition, the lower fascicle was more active than the upper fascicle during rest and most functional activities.

\section{REFERENCES}

1. World Health Organization. Ageing and life course. Available at: http://www who.int/topics/ageing/en/. (Accessed July 4, 2017.)

2. Partida MN. Geriatric prosthodontic care. Dent Clin North Am 2014;58 103-12.

3. Diz P, Scully C, Sanz M. Dental implants in the medically compromised patient. J Dent 2013;41:195-206.

4. Lynch CD, Allen PF. Overcoming the unstable mandibular complete denture: The neutral zone impression technique. Dent Update 2006;33: 21-2, 4-6.

5. Ali SA, Karthigeyan S, Deivanai M, Kumar A. Implant rehabilitation for atrophic maxilla: A review. J Indian Prosthodont Soc 2014;14:196-207.

6. Darvell BW, Clark RK. The physical mechanisms of complete denture retention. Br Dent J 2000:189:248-52.

7. Goiato MC, Garcia AR, Dos Santos DM, Zuim PR. Analysis of masticatory cycle efficiency in complete denture wearers. J Prosthodont 2010;19:10-3.

8. Goiato MC, Bannwart LC, Moreno A, Dos Santos DM, Martini AP

Pereira LV. Quality of life and stimulus perception in patients rehabilitated with complete denture. J Oral Rehabil 2012;39:438-45.

9. Proctor GB. The physiology of salivary secretion. Periodontol 2000 2016;70: $11-25$.

10. Grimby G, Saltin B. The ageing muscle. Clin Physiol 1983;3:209-18.

11. Heft MW, Robinson ME. Age differences in orofacial sensory thresholds. J Dent Res 2010:89:1102-5.

12. Nakamura A, Yamada T, Goto A, Kato T, Ito K, Abe Y, et al. Somatosensory homunculus as drawn by MEG. Neuroimage 1998;7:377-86.

13. Santos CM, Vitti M, Mattos MdGC, Semprini M, Paranhos HdFO, Hallak JEC, et al. Effect of denture quality on perioral muscle activity during speech. Braz J Oral Sci 2005;4:801-5.

14. Green JR, Moore CA, Higashikawa M, Steeve RW. The physiologic development of speech motor control: Lip and jaw coordination. J Speech Lang Hear Res 2000;43:239-55.

15. Park JS, Oh DH, Chang MY. Effect of expiratory muscle strength training on swallowing-related muscle strength in community-dwelling elderly individuals: A randomized controlled trial. Gerodontology 2017:34:121-8.

16. Regalo SC, Vitti M, Moraes MT, Semprini M, de Felicio CM, de Mattos Mda G, et al. Electromyographic analysis of the orbicularis oris muscle in oralized deaf individuals. Braz Dent J 2005:16:237-42.
17. Goiato MC, Garcia AR, dos Santos DM. Electromyographic activity of the mandible muscles at the beginning and end of masticatory cycles in patients with complete dentures. Gerontology 2008;54:138-43.

18. Berretin-Felix G, Nary Filho H, Padovani CR, Trindade Junior AS, Machado WM. Electromyographic evaluation of mastication and swallowing in elderly individuals with mandibular fixed implant-supported prostheses. J Appl Oral Sci 2008;16:116-21.

19. Tosello DO, Vitti M, Berzin F. EMG activity of the orbicularis oris and mentalis muscles in children with malocclusion, incompetent lips and atyp ical swallowing-part II. J Oral Rehabil 1999;26:644-9.

20. Castroflorio T, Falla D, Tartaglia GM, Sforza C, Deregibus A. Myoelectric manifestations of jaw elevator muscle fatioue and recovery in healthy and TMD subjects. J Oral Rehabil 2012;39:648-58.

21. Tulley WJ. Muscle and teeth. Proc R Soc Med 1957;50:313-20.

22. Baril C, Moyers RE. An electromyographic analysis of the temporalis muscles and certain facial muscles in thumb- and finger-sucking patients. J Dent Res 1960;39:536-53

23. Gamboa NA, Miralles R, Valenzuela S, Santander H, Cordova R, Bull R, et al. Comparison of muscle activity between subjects with or without lip competence: Electromyographic activity of lips, supra- and infrahyoid mus cles. Cranio 2016:1-7.

24. Santos CM, Vitti M, de Mattos Mda G, Semprini M, Paranhos Hde F, Regalo SC. Electromyographic analysis of the upper and lower fascicles of the orbicular oris muscle, in edentulous patients, before and after complete denture implantation. Electromyogr Clin Neurophysiol 2003:43:315-20.

25. Ingervall B, Hedegard B. An electromyographic study of masticatory and lip muscle function in patients with complete dentures. J Prosthet Dent 1980;43: 266-71.

26. Stal P, Eriksson PO, Eriksson A, Thornell LE. Enzyme-histochemical and morphological characteristics of muscle fiber types in the human buccinator and orbicularis oris. Arch Oral Biol 1990;35:449-58.

27. Shiffman MA, Di Giuseppe A. Cosmetic surgery: Arts and techniques. 15th ed. Berlin: Springer; 2012. p. 29-45.

28. American College of Prosthodontists. Prosthodontic Diagnostic Index (PDI) classification system. Prosthodontic Diagnostic Index Complete Edentulism Checklist. Available at: https://www.prosthodontics.org/assets/1/7/ Complete_Edentulism_Checklist.pdf.

29. American Society of Anesthesiologists. ASA physical status classification system. Available at: https://www.asahq.org/resources/clinical-information/ asa-physical-status-classification-system. (Accessed July 4, 2017.)

30. Dworkin SF, LeResche L. Research diagnostic criteria for temporomandibular disorders: Review, criteria, examinations and specifications, critique. J Craniomandib Disord 1992;6:301-55

31. Zuccolotto MC, Vitti M, Nobilo KA, Regalo SC, Siessere S, Bataglion C. Electromyographic evaluation of masseter and anterior temporalis muscles in rest position of edentulous patients with temporomandibular disorders, before and after using complete dentures with sliding plates. Gerodontology 2007; $24: 105-10$

32. Yagci A, Uysal T, Kara S, Okkesim S. The effects of myofunctional appliance treatment on the perioral and masticatory muscles in class II, division 1 patients. World J Orthod 2010;11:117-22.

33. Sonego MV, Goiato MC, Dos Santos DM. Electromyography evaluation of masseter and temporalis, bite force, and quality of life in elderly patients during the adaptation of mandibular implant-supported overdentures. Clin Oral Implants Res 2017:28:e169-74.

34. Zarb GA, Hobkirk J, Eckert S, Jacob R. Prosthodontic treatment for edentulous patients: complete dentures and implant-supported prostheses. 13th ed. St Louis: Elsevier/Mosby; 2013. p. 211-426.

35. Goiato MC, Ribeiro Pdo P, Garcia AR, dos Santos DM. Complete denture masticatory efficiency: A literature review. J Calif Dent Assoc 2008;36: 683-6.

Corresponding author:

Dr Marcelo Coelho Goiato

Aracatuba Dental School (São Paulo State University)

Department of Dental Materials and Prosthodontics

José Bonifácio, 1193 Vila Mendonca

16015-050 Aracatuba, São Paulo

BRAZIL

Email: goiato@foa.unesp.br

Copyright (C) 2017 by the Editorial Council for The Journal of Prosthetic Dentistry. 